\title{
Fast Sampling of Adherent Cell Cultures for Optimal Metabolomics Results

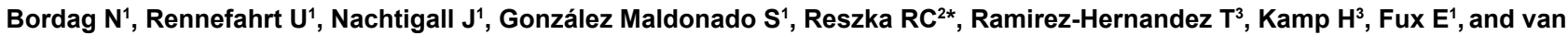 Ravenzwaay $\mathrm{B}^{3}$ \\ ${ }^{1}$ metanomics $\mathrm{GmbH}$, Berlin, Germany \\ ${ }^{2}$ Metanomics Health $\mathrm{GmbH}$, Berlin, Germany \\ ${ }^{3}$ BASF SE, Ludwigshafen, Germany
}

\begin{abstract}
Metabolomics is a valuable tool to gain mechanistic insight into biological processes. It is frequently used to obtain complementary details to other 'omics technologies such as transcriptomics or proteomics. For knowledge generation, reproducible measurements of physiological, intact, and artifact-free metabolite levels are imperative necessitating further standardization of best practices to improve reliability of research outcomes. Here we report a novel cell sample preparation method (MxP ${ }^{\circledR}$ CellCollect) for metabolomics applications using adherent mammalian cells, which reduces the time consumption and physiological stress of conventional methods such as trypsinization or cell scraping. The most common sampling procedures to detach cells from their growth surface, trypsinization and scraping were compared to the MxP ${ }^{\circledR}$ CellCollect method investigating metabolite profiles of two breast cancer cell lines (MDA-MB-231 and MCF7). Metabolite levels as well as direction of metabolite changes differed tremendously revealing issues with trypsinization and scraping risking non-physiological or misleading results in contrast to $\mathrm{MxP}^{\circledR}$ CellCollect. Differences in metabolic profiles of cells harvested by trypsinization as compared to $M \times P^{\circledR}$ CellCollect or scraping can be directly attributed to prolonged, medium-free incubation time during cell detachment leading to a severely energy-depleted intracellular state. Labile metabolites or metabolites with fast intracellular turnover rates such as glycolysis and TCA cycle intermediates were strongly and significantly decreased by trypsinization. The same was true for amino acids and nucleoside triphosphates. Results obtained with scraping using methanol as solvent were multifaceted. Even mild evaporation of methanol prior metabolite extraction led to temperature- and/or light-dependent degradation of labile metabolites such as nucleoside triphosphates into di- and monophosphates liberating pyrophosphate. Furthermore, lipid metabolites, in particular cell membrane lipids, were found to have significantly lower levels than measured by trypsinization or MxP CellCollect, indicating that lipid metabolites are insufficiently detached and/or unspecifically adsorb to the hydrophobic dish and scraping tool.
\end{abstract}

Keywords: Metabolomics; In vitro sampling procedures; Adherent eukaryotic cells; Scraping; Trypsinization; $\mathrm{MxP}^{\circledR}$ CellCollect

\section{Abbreviations}

ANOVA: Analysis of Variance; $\mathrm{CHCl}_{3}$ : Chloroform; DCM: Dichloromethane; EtOH: Ethanol; FBS: Fetal Bovine Serum; GC: Gas Chromatography; HPLC: High Performance LC; ISTD: Internal Standard; LC: Liquid Chromatography; $\mathrm{MeOH}$ : Methanol; MRM: Multiple Reaction Monitoring; MS: Mass Spectrometry; PBS: Phosphate Buffered Saline; PCA: Principal Component Analysis; RPMI: Roswell Park Memorial Institute; TCA: Tricarboxylic Acid Cycle; TNBC: Triple Negative Breast Cancer; s: Second(s)

\section{Introduction}

Metabolomics is a valuable tool generating biological insight from the most diverse sample types ranging from plants, to plasma, tissue or cell culture. The use of metabolomics to obtain insight into the toxicological properties and mode of action of compounds when tested in rats, has been demonstrated in the MetaMap ${ }^{\circledR}$ Tox Project $[1,2]$ and a successful step towards using this technology for in vitro systems was shown by [3].With adherent cells metabolomics is now becoming an invaluable tool to study disease mechanisms and mode of action of compounds, to discover drug targets and off-target effects, to observe resistance strategies, and to investigate toxicities [4-12]. A suitable sampling method is a key feature for routine use of high quality metabolomics with adherent cells. The method must be sufficiently robust, reproducible, easily performable, and most importantly, able to capture the physiological, non-perturbed intracellular metabolism [7]. The purpose of this investigation was to compare the most commonly used sampling procedures such as trypsinization and scraping, with a newly developed method called $\mathrm{MxP}^{\circledR}$ CellCollect and to suggest better standards in metabolomics sampling practices.

\section{Materials and Method \\ Cell culture and harvesting}

Six technical replicates per cell line and harvest condition were cultivated. MDA-MB-231 cells were freshly obtained from ATCC (HTB-26, Lot. 59922846) using passage 8 and MCF7 house-kept cells equivalent to ATCC (HTB-22) using passage 11 for experiments. Cells harvested by trypsinization or scraping were grown in standard uncoated $8.70 \mathrm{~cm}^{2}$ 6-well polystyrene culture dishes (83.3920.300, Sarstedt AG\&Co.). Cells harvested by $\mathrm{MxP}^{\circledR}$ CellCollect were grown in uncoated $6.4 \mathrm{~cm}^{2}$ lumox ${ }^{\circledR}$ dishes 35 (94.6077.331, Sarstedt AG\&Co.). Per dish $0.25 \cdot 10^{6}$ MDA-MB-231 and $0.3 \cdot 10^{6}$ MCF7 cells were seeded and grown in RPMI-1640 medium $(+10 \% \mathrm{FBS}+2 \mathrm{mM}$ glutamine $+100 \mu \mathrm{g} / \mathrm{ml}$ Penicillin/Streptomycin) under standard growth conditions $\left(37^{\circ} \mathrm{C}, 5 \%\right.$ $\mathrm{CO}_{2}$ ) reaching $80 \%$ confluence after $48 \mathrm{~h}$.

For trypsinization, as described in [13], cells were quickly washed

*Corresponding author: Reszka RC, Metanomics Health $\mathrm{GmbH}$, Berlin Germany, Tel: +49 3034807 407; Fax: +49 3034807 401; E-mail: regina.reszka@ metanomics-health.de

Received January 06, 2016; Accepted January 23, 2016; Published January 25, 2016

Citation: Bordag N, Rennefahrt U, Nachtigall J, González Maldonado S, Reszka RC, et al. (2016) Fast Sampling of Adherent Cell Cultures for Optimal Metabolomics Results. Metabolomics 6: 164. doi:10.4172/2153-0769.1000164

Copyright: $\odot 2016$ Bordag N, et al. This is an open-access article distributed under the terms of the Creative Commons Attribution License, which permits unrestricted use, distribution, and reproduction in any medium, provided the original author and source are credited. 
once with PBS (HPLC grade) and $0.2 \mathrm{~mL} 0.05 \%$ trypsin in $0.02 \%$ EDTA (R-001-100, Gibco ${ }^{\circledR}$ ) were added for $5 \mathrm{~min}$ at $37^{\circ} \mathrm{C}$. Detached cells were gently suspended in $0.4 \mathrm{~mL}$ fresh medium $\left(37^{\circ} \mathrm{C}\right)$, transferred and spun down with $900 \mathrm{rpm}$ for $3 \mathrm{~min}$ at $4^{\circ} \mathrm{C}$. To mimic cell counting an artificial break of 5 min was introduced. Cells were thereafter washed twice with $\mathrm{PBS}\left(4^{\circ} \mathrm{C}\right)$.

For scraping, as described in [12], cell monolayers were quickly washed twice with ice-cold 5\% maltose solution and residual fluid was removed with vacuum. Immediately $1 \mathrm{~mL} \mathrm{MeOH} \mathrm{(HPLC}$ grade) $\left(-20^{\circ} \mathrm{C}\right)$ was added and cells were scraped (2-Posit. Blade 25 , thermoplastic elastomers, Sarstedt AG\&Co).

For $\mathrm{MxP}^{\circledR}$ CellCollect (Figure 1) the lumox ${ }^{\circledR}$ membrane (with the adherent cells on) was cut out with a scalpel, falling into a dish with $37^{\circ} \mathrm{C}$ warm washing buffer (isotonic $\mathrm{NaCl}$ for $\mathrm{MxP}^{\circledR}$ Broad Profiling or $4.5 \mathrm{~g} / \mathrm{L}$ glucose in isotonic $\mathrm{NaCl}$ for $\mathrm{MxP}^{\circledR}$ Energy) [14]. The membrane was quickly picked up with tweezers and dip-washed twice in two further dishes with fresh washing buffer. The membrane was placed on top of a $2 \mathrm{~mL}$ Eppendorf tube and pushed inside with a pipette tip adding $600 \mu \mathrm{L} \mathrm{DCM} / \mathrm{EtOH}$ (9:11) for $\mathrm{MxP}^{\circledR}$ Broad Profiling or $900 \mu \mathrm{L}$ (2:1) $\mathrm{DCM} / \mathrm{EtOH}$ for $\mathrm{MxP}^{\circledR}$ Energy $\left(-80^{\circ} \mathrm{C}\right)$. The procedure from cutting the membrane until freezing should not exceed $30 \mathrm{~s}$ for each sample to prevent metabolic changes during sampling. All harvested cells were immediately snap frozen in liquid nitrogen and stored until extraction at $-80^{\circ} \mathrm{C}$. Directly before extraction, the $\mathrm{MeOH}$ of scraped cells was removed by mild evaporation over a few minutes at $40^{\circ} \mathrm{C}$ and $\mathrm{DCM} / \mathrm{EtOH}$ (mixtures as above) were added. Likewise, DCM/EtOH mixtures were added to trypsinized cell pellets after thawing before routine extraction and measurement (described in supplementary information).

\section{Statistical analysis}

Metabolite values were $\log 10$-transformed for all statistical analysis in order to better approximate a normal distribution. For univariate analysis a linear ANOVA model was set up in the statistical software $\mathrm{R}$ [15] with the categorical fixed factors METHOD and CELL_LINE (readouts: ratios, p-values, t-values, Benjamini-Hochberg corrected q-values in supplementary Table 1). For multivariate analysis, data was further centered and scaled to unit variance and multivariate statistics were performed by using Simca P+ software (version 13, Umetrics AB, Umea, Sweden).

\section{Results and Discussion}

A quick sampling is a key prerequisite in order to capture physiological intracellular metabolite levels and to avoid loss of metabolites with high turnover rates (e.g. glycolysis intermediates). Since metabolite concentrations are very sensitive to any variation in the cell environment an ideal harvest and quenching method for metabolomics analysis would immediately stop all intracellular

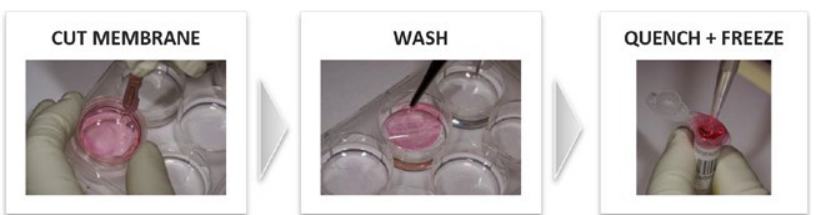

Figure 1: Sampling procedure. MxP ${ }^{\circledR}$ CellCollect sampling consists of cutting the membrane, dip washing the cells on the membrane, and quenching the metabolism with $-80^{\circ} \mathrm{C}$ cold solvent. The transparent membrane was colored red for better visibility. enzymatic activities and subsequently all intracellular changes in metabolite concentrations, without first affecting the cell environment. Furthermore, such a method should be rapid, highly efficient, and reproducible to allow an unbiased measurement of cellular metabolite concentrations, and to enable direct comparison of a large number of analytical samples. At the same time all cells with all of their metabolites must be collected and contaminations from spent media have to be avoided [16]. The necessary washing must leave metabolism unperturbed and should not induce any leakage or metabolite loss. Although great advances have been achieved by introduction of direct quenching and scraping instead of trypsinization [11,16-19] still a major drawback remains, namely the need for manual scraping and collection of cells. These steps might increase the variability of biological replicates due to differences in efficiency of cell transfer from the cell culture dish or cover slight into the extraction vial. Furthermore, these steps are time-consuming and therefore, might reduce overall attractiveness of this method to be used in high throughput cell culture experiments including several hundred samples in parallel.

\section{New sampling method $-\mathbf{M x P}^{\circledR}$ CellCollect}

The cells are grown on lumox ${ }^{\circledR}$ dishes, which foremost offer excellent gas diffusion through the thin, gas-permeable, membranelike bottom on which the cells grow. More importantly, these dishes allow cutting out the membrane and thereby to collect all cells quickly at once (Figure 1). The cells on the membrane are gently and thoroughly washed by immediate dipping into two consecutive dishes. The metabolism is then rapidly quenched by pushing the membrane into a tube with a pipette tip, immediately adding $-80^{\circ} \mathrm{C}$ precooled solvent and further snap freezing in liquid nitrogen. The time from cells being without medium until quenching is typically around a few seconds and the whole procedure takes less than $30 \mathrm{~s}$. Standard coatings such as fibronectin were well compatible and cell-free blanks incubated with medium as additional controls showed no impact on results.

\section{Comparison of trypsinization and scraping}

The two most conventional cell harvesting protocols, trypsinization [13]. and scraping in cold $\mathrm{MeOH}$ [12], were directly compared to the developed method for two different cell lines while all other parameters (extraction, measurement, normalization) were kept constant. Both multivariate (PCA, supplementary information Figure S1) and univariate analysis (ANOVA, Figure 2 and supplementary information Table 1) revealed obvious differences between all three methods. Differences in the metabolic profiles of cells harvested by trypsinization as compared to $\mathrm{MxP}^{\circledR}$ CellCollect or scraping can be directly attributed to the prolonged, medium-free incubation time during cell detachment leading to a severely energy-depleted intracellular state (Figure 2a). Glycolysis and TCA cycle intermediates as well as almost all amino acids and nucleoside triphosphates were strongly and statistically significantly decreased. Adenylate energy charge $(\mathrm{AEC}=\mathrm{ATP}+0.5 \cdot \mathrm{ADP} /$ $(\mathrm{ATP}+\mathrm{ADP}+\mathrm{AMP}))$ was with a value of $0.70( \pm 0.05)$ far below AEC levels found with scraping or $\mathrm{MxP}^{\circledR}$ CellCollect (with values of 0.82 $( \pm 0.07)$ and $0.84( \pm 0.03)$, respectively). Although trypsinization is a valuable method for cell detachment from their growth surface (e.g. for cell passaging), our results and literature data $[4,20]$ emphasize that trypsinization is not a suitable choice for physiological metabolomics since trypsin severely alters the physiological state of cells due to its interaction with membrane proteins resulting in sustained membrane damage in combination with metabolite leakage, and decreased cell viability, all of which significantly alter metabolite levels and cellular metabolite profiles [11]. As a consequence a considerable and highly 
a

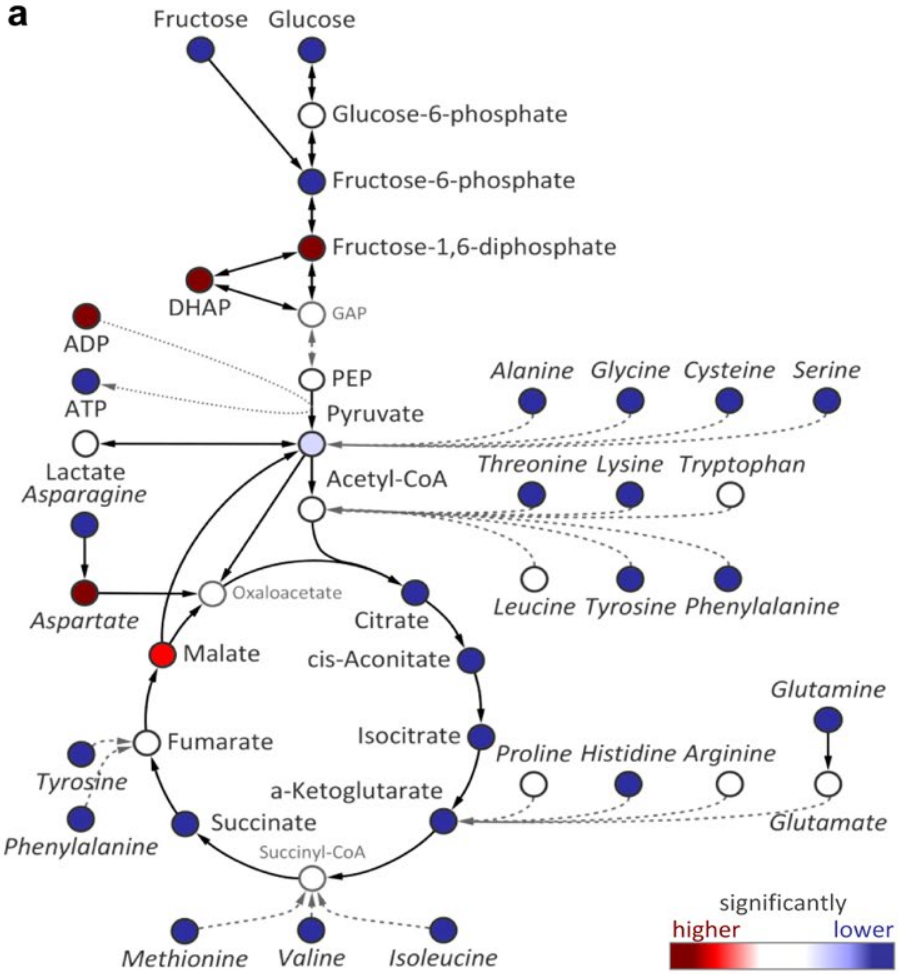

b

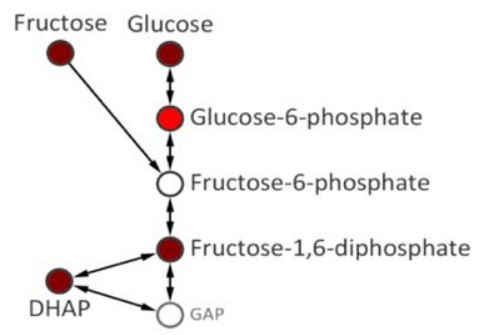

C

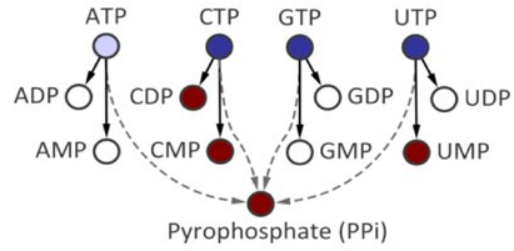

d

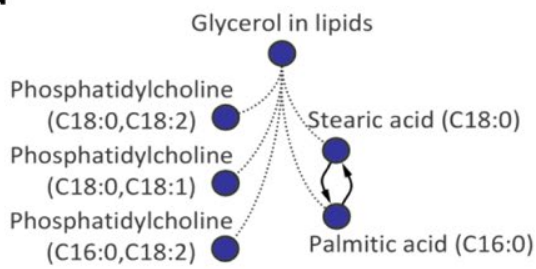

Figure 2: ANOVA comparison of different sampling methods across both cell lines. For a given comparison between two sampling methods red metabolite nodes signify higher metabolite levels while blue nodes signify lower levels with a significance of q-values $<0.1$ for least intensive colors to q-values $<0.01$ for most intensive colors. Grey metabolites were not measured and grey dashed arrows implicate several enzymatic reaction steps which were omitted for the sake of clarity. a) Comparison of trypsinization versus $\mathrm{MxP}^{\circledR}$ CellCollect reveals differences in glycolysis, TCA-cycle and proteinogenic amino acids. b), c), d) Comparison of scraping versus MxP ${ }^{\circledR}$ CellCollect with b) focusing on first steps of glycolysis, c) summarizing results for nucleoside tri-, di- and monophosphates and d showing results of selected lipid metabolites and their fatty acid precursors. The glycerol in lipids describes the sum of all glycerol moieties that is released from the lipid fraction of a biological sample after hydrolysis of complex lipids and subsequent derivatization prior to gas chromatographic analysis. Several phosphatidylcholines are shown as examples together with two saturated fatty acids which represent the sum of its occurrence both in free and in all lipid-bound forms (dotted lines indicate that fatty acids and phosphatidylcholines contribute to the total sum of conjugated glycerol in complex lipids).

variable portion of metabolites will be released into the trypsin solution and/or washing buffer depending on the length of trypsinization and washing/centrifugation steps. Furthermore, metabolic data varies for extremely labile metabolites or for metabolites with fast turnover rates in response to changes in cell environment and the numerous time-consuming steps (e.g. centrifugation) required before enzymatic activities are finally inhibited by cell quenching $[4,11]$. During this time, a considerable portion of intracellular metabolites is lost from cells due to their small molecular size, the intracellular fast turnover rates $[11,21]$, and and the different osmotic strength of the applied solutions $[22,23]$. Nevertheless, trypsinization was and is still used frequently $[13,24]$ and data obtained from trypsinized cells should be treated with utmost care, particularly if related to energy metabolism. The main reason for the use of trypsinization is the possibility to determine cell numbers for normalization purposes of the metabolic data. However, other normalization methods are available such as normalizing to cellular DNA after extraction [25]. In contrast, we have applied the median of all metabolites in a sample for normalization, which works well if the number of metabolites is sufficiently high $(>50)$ and data per sample is sufficiently comprehensive ( $<30 \%$ missing values). Median normalization also saves considerable time and resources because additional measurements such as DNA or additional biological replicates for cell counting can be omitted. Additionally, harsh extraction protocols with mechanical cell disruption (e.g. bead milling) are necessary for good overall metabolite recoveries, in particular for recovery of lipid metabolites [17], but also strongly fragmented cellular DNA rendering DNA normalization impossible.

Results obtained with scraping were multifaceted and three major artificial influences were identified. First ${ }_{2}$ the chosen protocol from Urakami et al. included a washing step with $5 \%$ maltose which led, despite the very short incubation time, to a considerable increase in early glycolysis intermediates (Figure $2 b$ ). This emphasizes the importance of choosing adequate washing solutions. Preferably noncold, isotonic, phosphate-free solutions should be used containing the same carbon sources as the culture medium tested. These carbon sources can also be fully isotopically labeled, such as all ${ }^{13} \mathrm{C}$, thereby slowing down ${ }^{12} \mathrm{C}$ metabolism during washing and stabilizing early glycolysis intermediates (Bordag et al. in preparation). The second observed effect was that even mild evaporation of the scraping solvent $\mathrm{MeOH}$ led to temperature- and/or light-dependent degradation of labile metabolites such as nucleoside triphosphates into di- and monophosphates liberating pyrophosphate (Figure 2c). For $\mathrm{MeOH}-$ based extractions solvent removal is not necessary. However, the $\mathrm{MeOH}$ poses problems for other extractions and needs to be removed which would require validation of even milder solvent removal (e.g. by lowering temperature or reducing exposure to light or evaporation time). Third, levels of lipids especially from cell membrane lipids were found to have significantly lower levels than measured by trypsinization or $\mathrm{MxP}^{\circledR}$ CellCollect (Figure 2d). These findings indicate that lipid 
metabolites are insufficiently detached from the dish bottom and/or lipidic metabolites unspecifically adsorb to the hydrophobic dish and scraping spatula (area offered for adsorption is very large in comparison to the low amounts of lipidic metabolites). In order to achieve complete recovery of lipidic metabolites the use of harsher solvents for scraping is necessary which in turn might risk the dissolving of the cell culture dish polystyrene plastic. In contrast, $\mathrm{MxP}^{\circledR}$ CellCollect is compatible with many solvents and harsh mechanical cell disruption which is a prerequisite for high recovery of polar and apolar metabolites [17] from the complete sample.

\section{Conclusion}

We developed a user-friendly, fast, efficient, reproducible, and robust sampling method for adherent cells. In summary, $\mathrm{MxP}^{\circledR}$ CellCollect proves to deliver more reliable results for metabolic profiling of adherent cells than the widely used scraping and trypsinization procedures. The presented manual sampling is extremely flexible and easily adoptable. The method was developed so that it is also suitable for possible automatization and translation into 96-well format in the future. More than 150 different cell lines have been analyzed by applying $\mathrm{MxP}^{\circledR}$ CellCollect so far, indicating overall that this new method is compatible with any adherent cell type and any common extraction or measurement protocol (MS- or NMR-based), easily adoptable without the need for sophisticated equipment. Here a high-throughput, routine MS-based protocol was applied which is also commonly used for sample types such as suspension cells, plasma, blood, or urine, thereby leading to increasing comparability.

\section{Acknowledgement}

The authors sincerely thank all of their team members and colleagues for their constant support and fruitful discussions.

\section{References}

1. van Ravenzwaay B, Cunha GCP, Leibold E, Looser R, Mellert W, et al. (2007) The use of metabolomics for the discovery of new biomarkers of effect. Toxicol Lett 172: 21-28.

2. van Ravenzwaay B, Coelho-Palermo Cunha G, Fabian E, Herold M, Kamp H, et al. (2010) The use of metabolomics in cancer research. An Omics Perspective of Cancer. Springer.

3. Balcke G, Kolle S, Kamp H, Bethan B, Looser R, et al. (2011) Linking energy metabolism to dysfunctions in mitochondrial respiration - A metabolomics in vitro approach. Toxicology Letters 203: 200-209.

4. Halama A (2014) Metabolomics in cell culture--a strategy to study crucial metabolic pathways in cancer development and the response to treatment. Arch Biochem Biophys 564: 100-109.

5. Kamp H, Fabian E, Groeters S, Herold M, Krennrich G, et al. (2012) Application of in vivo metabolomics to preclinical/toxicological studies: case study on phenytoin-induced systemic toxicity. Bioanalysis 4: 2291-2301.

6. Kentner D, Martano G, Callon M, Chiquet P, Brodmann M, et al. (2014) Shigella reroutes host cell central metabolism to obtain high-flux nutrient supply for vigorous intracellular growth. Proc Natl Acad Sci USA 111: 9929-9934.
7. Leon Z Garcia-Canaveras JC Donato MT, Lahoz A (2013) Mammalian cell metabolomics: experimental design and sample preparation. Electrophoresis 34: $2762-2775$.

8. Mattes W, Davis K, Fabian E, Greenhaw J, Herold M, et al. (2014) Detection of hepatotoxicity potential with metabolite profiling (metabolomics) of rat plasma. Toxicol Lett 230: 467-478.

9. Mattes WB, Kamp HG, Fabian E, Herold M, Krennrich G, et al. (2013) Prediction of clinically relevant safety signals of nephrotoxicity through plasma metabolite profiling. Biomed Res Int.

10. Ser Z, Liu X, Tang NN, Locasale JW (2015) Extraction parameters for metabolomics from cultured cells. Anal Biochem 475: 22-28.

11. Teng Q, Huang WL, Collette TW, Ekman DR, Tan C (2009) A direct cel quenching method for cell-culture based metabolomics. Metabolomics 5: 199208.

12. Urakami K, Zangiacomi V, Yamaguchi K, Kusuhara M (2013) Impact of 2-deoxy-D-glucose on the target metabolome profile of a human endometrial cancer cell line. Biomed Res 34: 221-229.

13. Reitman ZJ, Jin G, Karoly ED, Spasojevic I, Yang J et al. (2011) Profiling the effects of isocitrate dehydrogenase 1 and 2 mutations on the cellular metabolome. Proc Natl Acad Sci USA 108: 3270-3275.

14. Daemen A, Peterson D, Sahu N, McCord R, Du X et al. (2015) Metabolite profiling stratifies pancreatic ductal adenocarcinomas into subtypes with distinct sensitivities to metabolic inhibitors. Proc Natl Acad Sci USA 112: E4410-E4417.

15. R Development Core Team R2R (2014) A language and environment for statistical computing.

16. Martano G, Delmotte N, Kiefer P, Christen P, Kentner D, et al. (2015) Fast sampling method for mammalian cell metabolic analyses using liquid chromatography-mass spectrometry. Nat Protoc 10: 1-11.

17. Danielsson AP, Moritz T, Mulder H, Spegel P (2010) Development and optimization of a metabolomic method for analysis of adherent cell cultures. Anal Biochem 404: 30-39.

18. Lorenz MA, Burant CF, Kennedy RT (2011) Reducing time and increasing sensitivity in sample preparation for adherent mammalian cell metabolomics. Anal Chem 83: 3406-3414.

19. Ritter JB, Genzel Y, Reichl U (2008) Simultaneous extraction of several metabolites of energy metabolism and related substances in mammalian cells: optimization using experimental design. Anal Biochem 373: 349-369.

20. Dettmer K, Nurnberger N, Kaspar H, Gruber MA, Almstetter MF, et al (2011) Metabolite extraction from adherently growing mammalian cells fo metabolomics studies: optimization of harvesting and extraction protocols. Anal Bioanal Chem 399: 1127-1139.

21. Villas-Boas SG, Hojer-Pedersen J, Akesson M, Smedsgaard J, Nielsen J (2005) Global metabolite analysis of yeast: evaluation of sample preparation methods. Yeast 22: 1155-1169.

22. Britten RJ, Mcclure FT (1962) Amino Acid Pool in Escherichia-Coli. Bacterio Rev. 26: 292-335.

23. Smeaton JR, Elliott WH (1967) Selective release of ribonuclease-inhibitor from Bacillus subtilis cells by cold shock treatment. Biochem Biophys Res Commun 26: 75-81.

24. Ghosh JC, Siegelin MD, Vaira V, Faversani A, Tavecchio M, et al. (2015) Adaptive mitochondrial reprogramming and resistance to PI3K therapy. J Natl Cancer Inst 107

25. Silva LP, Lorenzi PL, Purwaha P, Yong V, Hawke DH, et al. (2013) Measurement of DNA concentration as a normalization strategy for metabolomic data from adherent cell lines. Anal Chem 85: 9536-9542. 